\title{
EJNSO
}

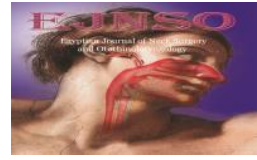

Factors affecting decannulation of elective tracheostomy done for

\section{patients in intensive care units}

M.M. Roushdy ${ }^{\mathrm{a}}$, H. S. Abdel-gaffar ${ }^{\mathrm{b}}$, A.E.Saleh ${ }^{\mathrm{a}}$, A. K. Abdel-haleem ${ }^{\mathrm{a}}$

a Departments of Otorhinolaryngology, ${ }^{\mathrm{b}}$ Anaesthesia and Intensive Care Units, Assuit University Hospital, Assuit, Egypt

Keywords: Intensive care, mechanical ventilation, elective tracheostomy, decannulation.

\begin{abstract}
Introduction: Prolonged intubation is the main indication for tracheostomy in intensive care units (ICUs). The aim of this study is to detect the factors affecting decannulation of tracheostomy done for patients in ICUs. Patients and Methods: It is a prospective cohort study. It was conducted in trauma ICUs of a tertiary care center. It included all patients who were admitted to ICUs during the period of the study and were subjected to prolonged intubation, mechanical ventilation and tracheostomy during their admission in ICUs, regardless of their age and sex. Decannulation was considered when no longer need for tracheostomy and after fulfilling criteria of decannulation. Decannulation was one staged and was considered successful if there was no need for reapplication of tracheostomy during six months of follow up. The participants were divided into two groups; successful decannulation group (DG) and failure of decannulation group (FDG). Factors affecting decannulation were detected by comparing the two groups; (DG) and (FDG). Results: DG included 52 patients and FDG included 29 patients. Tracheal stenosis and low conscious level were the cause of failure of decannulation in $44.8 \%$ and $41.4 \%$ of patients respectively. Statistically significant shorter duration of intubation and duration of mechanical ventilation were found in DG with p. value 0.015 and 0.025 respectively. The mean cannulation time of DG group was 49 days. Conclusion: Duration of intubation, duration of mechanical ventilation, development of tracheal stenosis, low conscious level and bad chest condition are the factors ruling decannulation of tracheostomy.
\end{abstract}

\section{Introduction:}

Prolonged endotracheal intubation in intensive care units (ICUs) can lead to serious laryngeal and tracheal injuries. ${ }^{1}$ Laryngeal edema, ulcerations, intubation granuloma, impairment of vocal cords mobility, laryngeal and tracheal stenosis are complications of prolonged endotracheal intubation. ${ }^{1,2}$ Tracheostomy in ICUs is an elective procedure to gain a surgical airway to trachea to replace endotracheal tubes in conduction of mechanical ventilation(MV)in prolonged ventilated patients. $^{3}$ It is performed frequently in intensive care unit (ICU). ${ }^{4}$ 
It protects larynx and upper airway from complications of prolonged translaryngeal intubation, It helps in suction of secretions from the airway. ${ }^{5}$ Furthermore, it helps in decreasing the incidence of pneumonia associated with mechanical ventilation. ${ }^{6}$ It decreases the amount of sedation required for patients in ICUs as well as increases patient's comfort, it also decreases dead space and airway resistance. Thus, decreases the duration of MV and ICU stay. 7-9 Tracheostomy provides a stable airway in critically ill patients in need for prolonged mechanical ventilation. ${ }^{9}$

Removal of tracheostomy tube "Decannulation" is indicated when the patient is not in need for tracheostomy tube anymore and when he/she fulfills the criteria of decannulation; (Patient is alert, responds to commands, not in need for assisted ventilation, Peripheral arterial oxygen saturation $\mathrm{SaO} 2 \%$ more than $92 \%$, no need for frequent tracheal suctioning, and can protect his/her airway with an effective cough reflex). Evaluation of the airway -using flexible endoscope- should be done to exclude laryngeotracheal stenosis or vocal cord paralysis. $^{10-14}$ Decannulation is a challenging multi factorial process. ${ }^{11,15}$ This study was conducted to detect causes and factors affecting decannulation of tracheostomy in patients of ICUs.

\section{Patients and Methods}

Ethical considerations: This prospective randomized cohort study was done after obtaining an institutional review board IRB approval from the medical ethics committee. (Approval date: 20/1/2016 and issued IRB no: 00008718). It was conducted in the Ear, Nose and Throat (ENT) department and trauma intensive care units (ICUs) of a tertiary care center during the period from 1st of June 2015 to 31st of May 2016 and it was registered in ClinicalTrials.gov and issued registration number (NCT 03431389). Before including participants in the study, the purpose and nature of the study were explained to their guardians as all our participants were critically ill patients and incapacitated and a written informed consent was obtained from all study participants' guardians.

All patients who were admitted to the trauma ICUs of tertiary care center and were mechanically ventilated and underwent tracheostomy during his/her stay in ICU were included in the study. Both sexes and all age groups were included. Patients who were admitted to ICUs and didn't need tracheostomy during the period of admission in ICU, died during admission in ICUs before being tracheostomized, died during admission in ICUs after being tracheostomized and the cause of death was not related to the tracheostomy procedure or care were excluded from the study.

Tracheostomy was done to all patients by the open surgical technique and was done in the ICUs without transfer to the operative theatre. The patients were followed up daily until discharge from ICU.

Decannulation was considered when the patients were no longer in need for tracheostomy tube and fulfilled the criteria of decannulation; (no need for mechanical ventilation, no chocking with oral intake, no chest infection, effective cough reflex, laryngeal examination shows bilateral mobile vocal cords with a sufficient gap. Decannulation was single staged and was done in ENT department and was done in the morning under close observation of the patient. 
Decannulation was done using a piece of gauze to occlude the tracheostomy stoma after removal of tracheostomy tube. Trial of decannulation was considered successful, if there was no need to reapply tracheostomy within 6 months of decannulation. Decannulation trail was considered failed if there was a need to reapplication of tracheostomy at the time of decannulation or within six months of decannulation the duration of follow up. Afterwards, patients were divided into two groups; Group of successful decannulation (DG) and group of failure of decannulation (FDG). Analysis of the two groups was done to detect the factors affecting successful decannulation.

Assessment parameters included; Age and sex, cause of admission in ICUs, duration of endotracheal intubation before performing tracheostomy. Duration of application of tracheostomy tube before decannulation (cannulation time), development of tracheal stenosis, conscious level of the patients upon discharge from ICUs, co morbidities that make the patients bedridden and complications of tracheostomy were recorded.

\section{Statistical analysis:}

The data were tested for normality using the Kolmogorov-Smirnov test and for homogeneity variances prior to further statistical analysis. Categorical variables were described by number and percent $(\mathrm{N}, \%)$, where continuous variables were described by mean and standard deviation [Mean (SD)]. Chisquare and fisher exact tests were used to compare between categorical variables. Unpaired t-test was used to compare between continuous variables for normally distributed data and Mann Whitney $U$ for non-normally distributed data. A two-tailed $\mathrm{P}<0.05$ was considered statistically significant. All analyses were performed with the IBM SPSS 20.0 software.

\section{Results}

From 1st of June 2015 to 31st of May 2016, two hundred and thirty-seven consecutive intubated and mechanically ventilated ICU patients were screened for eligibility. Ten patients were lost during the period of data recording either due to discharge upon request from the hospital or transfer to other ICUs, eighty-seven patients didn't undergo tracheostomy either during admission in ICUs and fifty-nine patients died during follow up in ICUs after being tracheostomized. Finally, 81 patients were successfully enrolled and subjected to statistical analysis and were further classified to two groups; Decannulated group (DG, $n=52$ ) and failure of decannulation group (FDG, $n=29)$, (Figure: 1).

Decannulation was achieved in $(52 / 81)$ patients $(64.2 \%)$, while failure of decannulation was recorded in (29/81) patients $(35.8 \%)$.

The mean age of DG was 21.85 years, while that of FDG was 26.1 years. In both groups, males were more than females. Males represented $80.76 \%$ and $89 \%$ in DG and FDG respectively. Forty-three patients $(82.69 \%)$ of DG were patients with head trauma while 22 patients $(75.68 \%)$ were head trauma patients in FDG. There was no statistically significant difference between the two groups regarding age, sex and cause of admission.

The duration of endotracheal intubation of DG was found to be statistically significant shorter compared to FDG. (7.88 \pm 3.3 for DG VS $10.1 \pm 4.66$ for FDG with ( $\mathrm{p}$ value 0.015 ) (Figure 2 and Table 1). The duration of mechanical ventilation of DG group was 
statistically significant shorter compared to FDG $(11.6 \pm 6.6$ for $\mathrm{DG}$ and $15 \pm 9.9$ for FDG with p. value 0.029).

(Figure 3 and table 1) Tracheal stenosis was diagnosed in 7 patients of DG compared to 13 patients of FDG (P. value 0.002). In DG there was no patients discharged from ICUs with low conscious level with Glasgow coma scale (GCS)below 8 compared to 12 patients in FDG (P value 0.0001). There were no bedridden patients in DG, while FDG included 4 patients who were bedridden ( $\mathrm{P}$ value 0.0065 ).

Early tracheostomy with short duration of intubation less than eight days was associated with successful decannulation. Also, the short duration of mechanical ventilation below 12 day associated with successful decannulation. Development of tracheal stenosis, low GCS below eight and being bedridden were found to be statistically associated with failure of decannulation.

Regarding complication of tracheostomy, tracheostomy stoma wound sepsis was the most common complication of tracheostomy and occurred in seven patients. Complications of tracheostomy summarized in (Table 2).

The mean duration of application of tracheostomy tubes (cannulation time) before successful decannulation was 49 days and ranged from 4-177 days, (Figure 4).

Tracheal stenosis was the cause of failure of decannulation in $44.8 \%$ $(13 / 29)$ of the patients. Poor neurological status (vegetative) with low GCS below eight and with persistent nasogastric tube feeding was the 2nd leading cause of failure of decannulation in $41.4 \%$ (12/29) patients. They had copious chest secretions and were not able to expectorate their own secretions and needed suction through tracheostomy. Bad chest condition due to persistent bedridden patients was the cause of failure of decannulation in $13.7 \%$ (4/29) patients. 


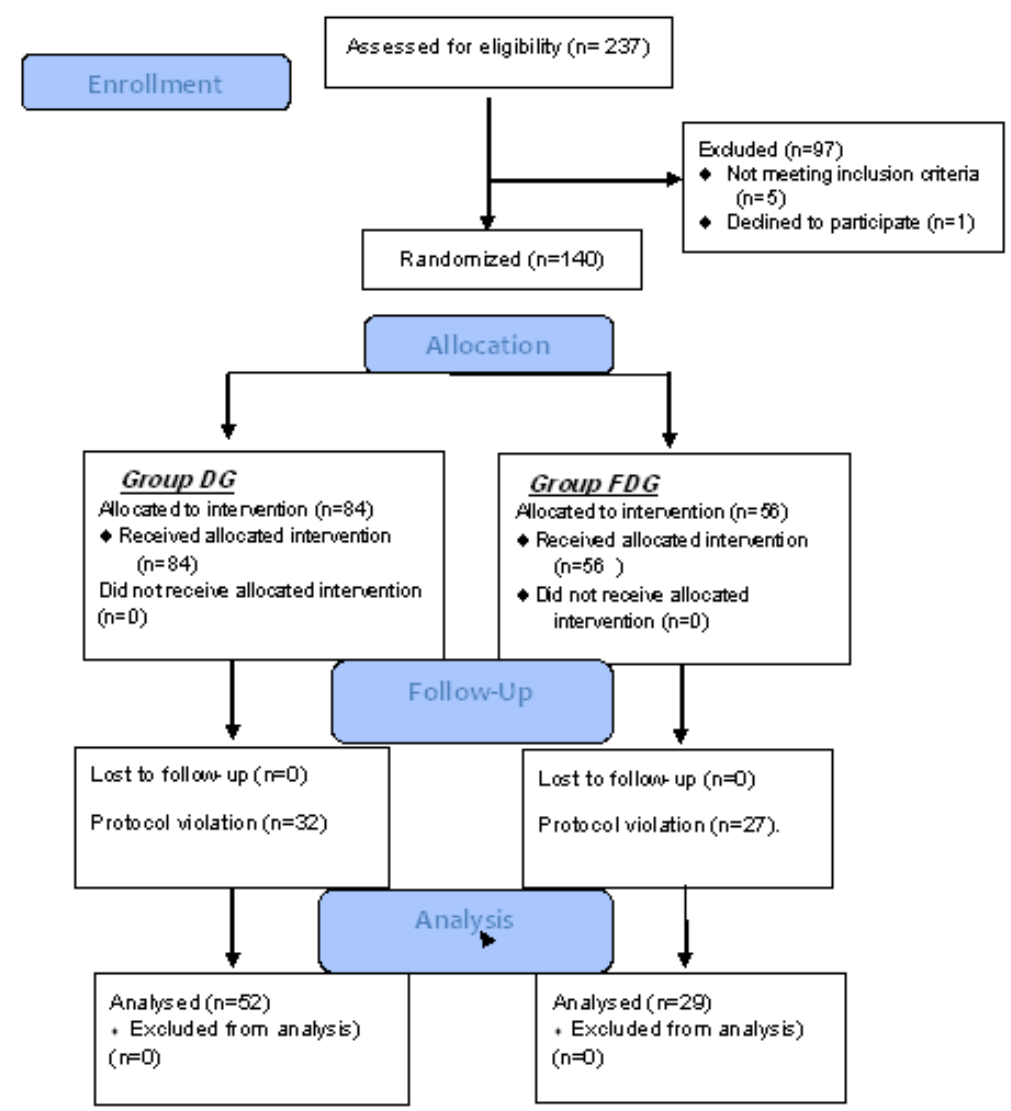

Figure 1: Participant Flow diagram. 
Table 1 Correlation between decannulation and clinical and demographic data

Data represented as mean $\pm \mathrm{SD}$, (n) numbers and $(\%)$ percentage, $* \mathrm{P}<0.05$

\begin{tabular}{|c|c|c|c|c|c|}
\hline & \multicolumn{4}{|c|}{ Decannulation } & \multirow{3}{*}{ P. value } \\
\hline & \multicolumn{2}{|c|}{$\begin{array}{c}\text { Successful } \\
\text { decannulation } \\
\quad(n=52)\end{array}$} & \multicolumn{2}{|c|}{$\begin{array}{c}\text { Failure of } \\
\text { decannulation } \\
(n=29)\end{array}$} & \\
\hline & No. & $\%$ & No. & $\%$ & \\
\hline Age, mean $\pm S D$ (in years) & \multicolumn{2}{|c|}{$21.94 \pm 15.35$} & \multicolumn{2}{|c|}{$25.3 \pm 18.15$} & 0.493 \\
\hline \multicolumn{6}{|l|}{ Sex } \\
\hline Male & 42 & 80.8 & 26 & 89.7 & \multirow{2}{*}{0.298} \\
\hline Female & 10 & 19.2 & 3 & 10.3 & \\
\hline \multicolumn{6}{|l|}{ Diagnosis of admission } \\
\hline Head trauma with brain injury & 43 & 82.7 & 22 & 75.8 & 0.462 \\
\hline Non-head trauma & 9 & 27.3 & 7 & 24.2 & 0.266 \\
\hline Duration of intubation (in days) * & \multicolumn{2}{|c|}{$\underline{7.9 \pm 3.52}$} & \multicolumn{2}{|c|}{$\underline{10.7 \pm 7.14}$} & $\underline{0.020 *}$ \\
\hline $\begin{array}{l}\text { Total duration of mechanical } \\
\underline{\text { ventilation (in days) * }}\end{array}$ & \multicolumn{2}{|c|}{$\underline{11.7 \pm 6.59}$} & \multicolumn{2}{|c|}{$\underline{17 \pm 10.3}$} & $\underline{0.006 *}$ \\
\hline $\begin{array}{l}\text { Ptients with low conscious level } \\
\underline{(\text { GCS below } 8) *}\end{array}$ & $\underline{0}$ & $\underline{0}$ & $\underline{12}$ & $\underline{41.4}$ & $\underline{0.0001 *}$ \\
\hline$\underline{\text { Bedridden patients* }}$ & $\underline{0}$ & $\underline{0}$ & $\underline{4}$ & $\underline{13.7}$ & $\underline{0.0065 *}$ \\
\hline Patients with tracheal stenosis* & $\underline{7}$ & $\underline{13.4}$ & $\underline{13}$ & $\underline{44.8}$ & $\underline{0.002 *}$ \\
\hline
\end{tabular}

significant difference between two groups 


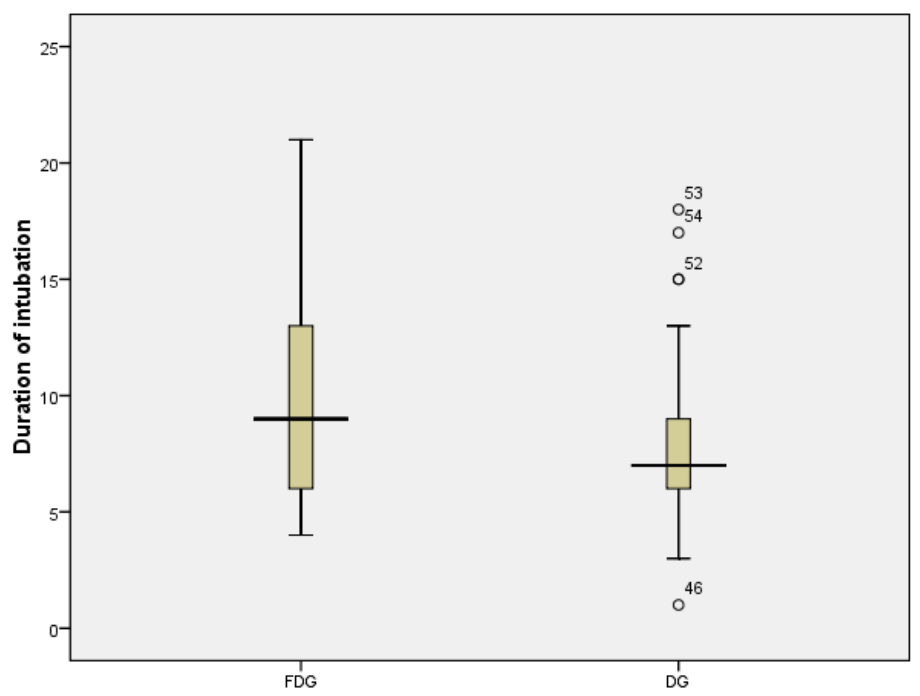

Decannulation group (DG) vs faillure of decanullation group (FDG)

Fig.2: Duration of intubation in two groups.

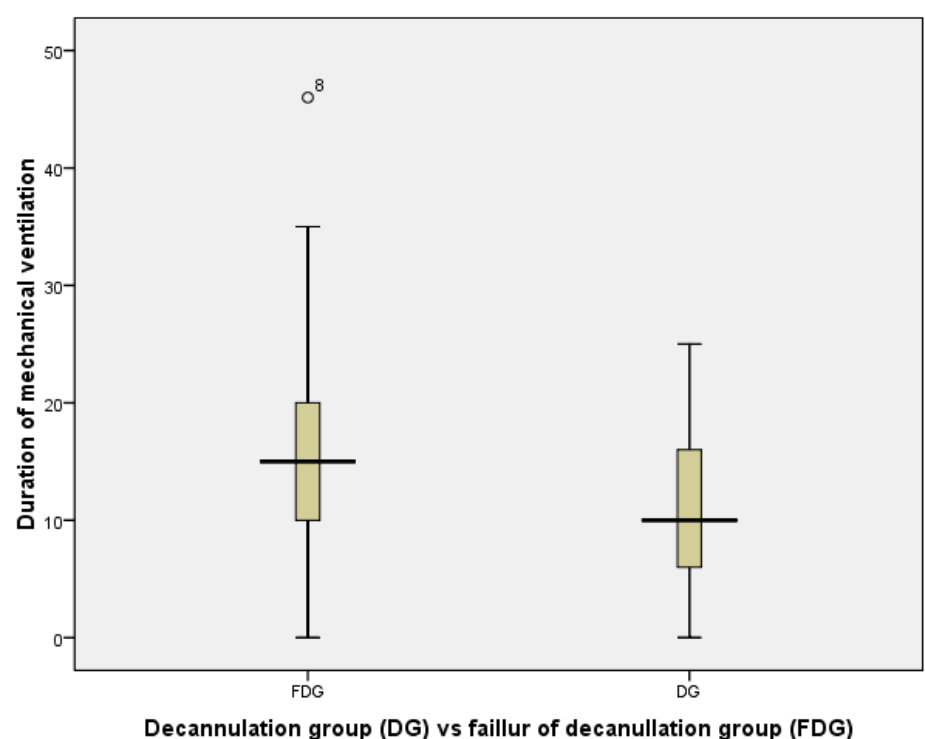

Figure 3: Duration of mechanical ventilation. 
Table 2 Complications of tracheostomy

\begin{tabular}{|l|c|c|}
\hline \multicolumn{1}{|c|}{ Complications } & $\begin{array}{c}\text { Number } \\
\text { of } \\
\text { patients } \\
\text { (n) }\end{array}$ & Percentage \\
$\mathbf{( \% )}$
\end{tabular}

Data represented as (n) numbers and (\%) percentage

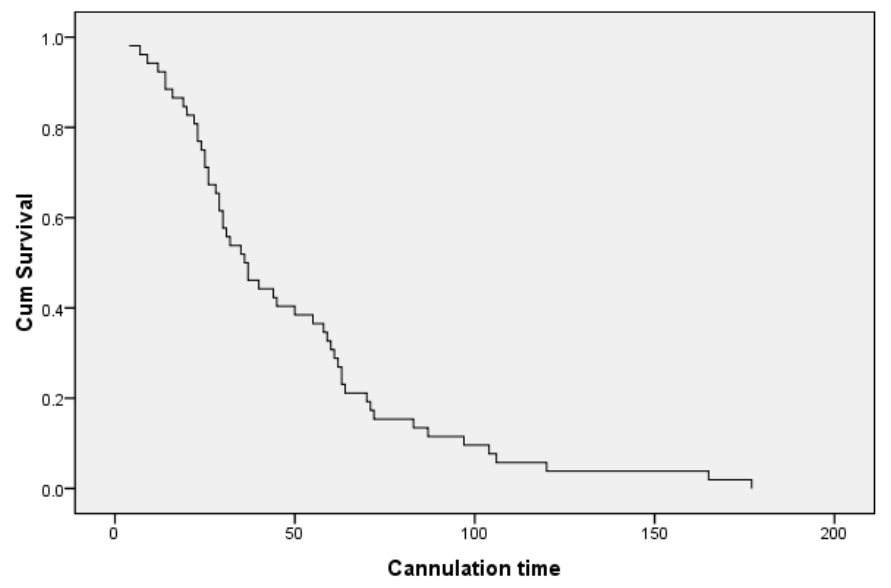

Figure 4: Cannulation time. 


\section{Discussion}

\section{Synopsis of key/new findings}

This study investigated the factors ruling decannulation of tracheostomy in patient subjected to tracheostomy due to prolonged mechanical ventilation in ICUs, its attributed failure of decannulation to prolonged endotracheal intubation, prolonged duration of $\mathrm{MV}$, low conscious level, development of tracheal stenosis and bed redden patients.

\section{Strengths of the study}

The fact that about $(75 \%)$ of the patients in this study were admitted to ICU because of trauma, notably with serious brain insults, markedly affected not only the demography of the patients but also most of the results regarding rate of tracheostomy in ICU and outcomes of tracheostomy in ICU patients. Most of the available literatures discussing items related to tracheostomy in ICUs reported causes of admission to ICUs other than trauma. $10,11,13,14,16,17$ However, the data available about trauma patients with head injury in ICUs were few or sporadic among other causes of admission in ICUs. ${ }^{18-20}$

\section{Comparisons with other studies}

In this study males were more than females with percentages $82.9 \%$ and $17.1 \%$ respectively, with male to female ration 4.8:1. Sex and age of the patients were found not to be affecting decannulation. These results were close to El-Anwar et al. (2017), Hydri et al. (2017) and Olton et al. (2009) who studied the tracheostomy in ICUs and recorded more males in their studies with $87.1 \%, \quad 78.5 \%$ and $68.8 \%$ respectively. $16,21,22$

Decannulation is an important step for critically ill patients to return to their normal life. The successful decannulation rate of this study $(64.2 \%)$ was higher than that of Leung and colleagues in 2005 who had $56.6 \%$ of their patients successfully decannulated. 10

It was proved in this study that the main factors affecting the success or failure of decannulation were; the timing of tracheostomy, duration of mechanical ventilation, the neurological and chest conditions of the patient, and the development of post intubation tracheal stenosis. Early tracheostomy directly allows early weaning from $\mathrm{MV}$ and indirectly decreases the development of tracheal stenosis, thus improves the chances for successful decannulation. Failure of decannulation due to poor neurological was the cause of failure in $41.4 \%(12 / 29)$ of the patients who failed decannulation. these results were inconsistence with stelfox et al. (2008) who found that patients conscious level was determinant for decannulation. ${ }^{15}$ These results were close to a study done in Australia in 2009, where retention of sputum and bad chest condition represented $52.5 \%$ of causes of failure of decannulation. ${ }^{23}$ In consistence with our results, Hydri et al. (2017) stated that decannulation was affected by patient's co morbidities. ${ }^{16}$

In spite of all the efforts that were done to the studied patients with brain injury, 12 patients had low GCS below eight until the end of this study and they were in need for tracheostomy. Four patients were bed redden, unable to expectorate effectively, remained in need for frequent suction of copious viscid pulmonary secretions. Here a question rises; would these patients be successfully decannulated if the study period was longer?

Tracheal stenosis that was diagnosed in 21 patients as cicatrized stenosis by rigid bronchoscopy, was an important determinant for decannulation. Failure of 
decannulation documented in $13(62 \%)$ of them.

The mean cannulation time in this study was 49 days with $57.7 \%$ of studied patients were decannulated within one month and only 3 patients needed more than 100 day to be decannulated.

Results regarding cannulation time were close to Shrestha et al. (2012) who studied patients with head injuries and had $58.5 \%$ of their patients were decannulated after one month, ${ }^{67}$ but longer than a study was conducted in Canada in 2010 and a study conducted in Australia in 2010. In these studies the recorded cannulation time were 41.9 days and 25 days respectively. ${ }^{24}$ The long cannulation time in our study can be attributed to the type of patients; they were trauma patients with serious brain injuries which require long recovery period after weaning from MV, also lack of definite protocol of decannulation and specific personnel responsible for decannulation process could be from the causes of long cannulation time. A study was conducted in Canada in 2010 to detect outcomes of tracheostomy in patients with traumatic brain injury following implementation of a specialized multidisciplinary tracheostomy team and compared the results before and after implementation of the tracheostomy team; the cannulation time was 6 days shorter after implementation of tracheostomy team. ${ }^{24}$ Leung and colleagues in 2005 had longer cannulation time 123 days in their study in which they studied decannulation in pediatric population.

\section{Limitations and clinical applicability of the study}

Limitations that were met during the study are marked variability of the age that affected the mean and standard deviation and most of our patents were trauma patients.

\section{$\underline{\text { References }}$}

1.Santos PM, Afrassiabi A, Weymuller EA, Jr. Risk factors associated with prolonged intubation and laryngeal injury. Otolaryngol Head Neck Surg. 1994;111(4):453-9.

2.De S, De S. Post intubation tracheal stenosis. Indian Journal of Critical Care Medicine: Peer-reviewed, Official Publication of Indian Society of Critical Care Medicine. 2008;12(4):194-7.

3.Paul J, Pracy M, Rogers M. Tracheostomy. fifth ed. New york Taylor \& Francis; 2012.

4.Aissaoui Y, Azendour H, Balkhi H, Haimeur C, Kamili Drissi N, Atmani M. Timing of tracheostomy and outcome of patients requiring mechanical ventilation. Ann Fr Anesth Reanim. 2007;26.

5.Alía I, Esteban A. Weaning from mechanical ventilation. Critical Care. 2000;4(2):72-80.

6.Durbin CG, Jr. Indications for and timing of tracheostomy. Respir Care. 2005;50(4):483-7.

7.Pierson DJ. Tracheostomy and weaning. Respir Care. 2005;50(4):52633.

8.Durbin. Tracheostomy: Why, When, and How? Respir Care Clin N Am. 2010.

9.Nieszkowska A, Combes A, Luyt CE, Ksibi H, Trouillet JL, Gibert C, et al. Impact of tracheotomy on sedative administration, sedation level, and comfort of mechanically ventilated intensive care unit patients. Crit Care Med. 2005;33(11):2527-33.

10.Leung R, MacGregor L, Campbell D, Berkowitz RG. Decannulation and survival following tracheostomy in an intensive care unit. Ann Otol Rhinol Laryngol. 2003;112(10):853-8.

11.Leung R, Berkowitz RG. Decannulation and outcome following pediatric tracheostomy. Ann Otol Rhinol Laryngol. 2005;114(10):743-8. 
12.Kontzoglou G, Petropoulos I, Noussios G, Skouras A, Benis N, Karagiannidis K. Decannulation in children after long-term tracheostomy. B-ENT. 2006;2(1):13-5.

13.O'Connor HH, Kirby KJ, Terrin N, Hill NS, White AC. Decannulation following tracheostomy for prolonged mechanical ventilation. J Intensive Care Med. 2009;24(3):187-94.

14.O'Connor HH, White AC. Tracheostomy decannulation. Respir Care. 2010;55(8):1076-81.

15.Stelfox HT, Crimi C, Berra L, Noto A, Schmidt U, Bigatello LM, et al. Determinants of tracheostomy decannulation: an international survey. Crit Care. 2008;12(1):R26.

16.Hydri As, Saeed I, Seddiqui F. Outcomes of surgical tracheostomies in an intensive care units. Rawal Medical Journal 2017;42.

17.Lim C-K, Ruan S-Y, Lin F-C, Wu CL, Chang H-T, Jerng J-S, et al. Effect of Tracheostomy on Weaning Parameters in Difficult-to-Wean Mechanically Ventilated Patients: A Prospective Observational Study. PLoS One. 2015;10(9):e0138294.

18.Dunham CM, Cutrona AF, Gruber BS, Calderon JE, Ransom KJ, Flowers LL. Early tracheostomy in severe traumatic brain injury: evidence for decreased mechanical ventilation and increased hospital mortality. International Journal of Burns and Trauma. 2014;4(1):14-24.

19.Rizk EB, Patel AS, Stetter CM, Chinchilli VM, Cockroft KM. Impact of tracheostomy timing on outcome after severe head injury. Neurocrit Care. 2011;15(3):481-9.

20.Bouderka MA, Fakhir B, Bouaggad A, Hmamouchi B, Hamoudi D, Harti A. Early tracheostomy versus prolonged endotracheal intubation in severe head injury. J Trauma. 2004;57(2):251-4.
21.El-Anwar MW, Nofal AA-F, Shawadfy MAE, Maaty A, Khazbak AO. Tracheostomy in the Intensive Care Unit: a University Hospital in a Developing Country Study. International Archives of Otorhinolaryngology. 2017;21(1):33-7.

22. Olton S, Hariharan S, Chen D. Outcome evaluation of patients requiring tracheostomy in an intensive care unit in Trinidad. West Indian Med J. 2009;58(2):173-8.

23.Choate K, Barbetti J, Currey J. Tracheostomy decannulation failure rate following critical illness: a prospective descriptive study. Aust Crit Care. 2009;22(1):8-15.

24.LeBlanc J, Shultz JR, Seresova A, de Guise E, Lamoureux J, Fong N, et al. Outcome in tracheostomized patients with severe traumatic brain injury following implementation of a specialized multidisciplinary tracheostomy team. J Head Trauma Rehabil. 2010;25(5):362-5. 